\title{
Automated Sectoral Quantitative Analysis of Parafoveal Microvascular Status and Association with Structure in Diabetic Macular Edema
}

Nihat Polat ( $\sim$ drnihatpolat@gmail.com )

Inonu University School of Medicine: Inonu Universitesi Tip fakultesi https://orcid.org/0000-00021735-1363

\section{Saim Yoloğlu}

Inonu University School of Medicine: Inonu Universitesi Tip fakultesi

\section{Research Article}

Keywords: Diabetic macular edema, parafoveal microvascular status, sectoral analysis, OCTA

Posted Date: June 25th, 2021

DOI: https://doi.org/10.21203/rs.3.rs-561485/v1

License: (c) (i) This work is licensed under a Creative Commons Attribution 4.0 International License.

Read Full License 


\section{Abstract}

Purpose: To evaluate the integrity of the capillary plexuses, their relationship with each other, and their association with structural changes in a sectoral manner in diabetic macular edema (DME).

Methods: The vessel density (VD) was measured in inferior, superior, nasal and temporal (i, S, N, T) parafovea in the superficial capillary plexus (SCP) and deep capillary plexus (DCP). The integrity of the external limiting membrane (ELM) and ellipsoid zone (EZ) were evaluated in B-scan images.

Results: In the SCP, only superior and nasal VD was significantly lower (P: 0.0001 and P: 0.020, respectively) while in the $D C P$, only temporal VD was significantly lower in the DME eyes (P: 0.034). There was no correlation between the VD of SCP and DCP in the corresponding quadrants (all P > 0.05). The FAZ area was significantly greater (both superficial and deep) in DME eyes than in control eyes $(P>0.05)$. ELM disruptions were not affected by the corresponding VDs in the SCP (all P>0.05), but nasal and temporal quadrant ELM disruptions were affected by VDs in the DCP in the corresponding quadrants (P: 0.005 nasal, $\mathrm{P}: 0.001$ temporal). EZ disruptions were not affected by the corresponding VDs in the SCP and DCP (all P>0.05).

Conclusions: The SCP was affected in more quadrants than the DCP and probably independently from each other. There was an association with the ELM and the DCP, especially in nasal and temporal quadrants, but there was not an association between the EZ and SCP or DCP.

\section{Introduction}

Diabetic macular edema (DME) is the most important cause of vision loss in patients with diabetic retinopathy (DR) $[1,2]$. DME is associated with a breakdown of the blood retinal barrier (BRB), and occlusion of retinal capillaries results in tissue hypoxia and leads to an increase in vascular endothelial growth factor and inflammatory factor levels, resulting in DME [3]. The inner retina has three capillary plexuses (the superficial capillary plexus (SCP), middle capillary plexus (MCP) and deep capillary plexus (DCP)), which are connected with anastomosis, but the majority of software for optical coherence tomography angiography (OCTA) has been limited to analyzing the SCP and DCP [4]. Previous studies have shown that microvascular alterations in individual capillary plexuses are associated with the severity of DR and DME [5-7]. Diabetic macular ischemia (DMI) is described as enlargement of the foveal avascular zone (FAZ) and the presence of capillary nonperfusion in the macular region $[3,8]$. DME often occurs as cystic form. Cystoid spaces mainly settle in the inner nuclear layer (INL) and outer plexiform layer (OPL) and occur because of intracytoplasmic swelling of Müller cells $[9,10]$. The possibility of macular ischemia increases when the diameter of the cyst increases, and DMI may cause further deterioration of vision [11]. Cystic spaces that are due to DME may distort the adjacent retinal layers and disrupt the normal foveal contour [12]. There are many studies that investigated the vascular status of the macula in DME, but it is unclear whether the change in vascular status is uniform in each parafoveal quadrant or differs. Some researchers have shown that diabetic capillary nonperfusion can be associated 
with photoreceptors and outer retinal disruption $[4,13,14]$. The authors have implicated the importance of vascular status and the association of the macular structure, but the sectoral association in this relationship is still unclear.

OCTA is a relatively new technology that provides a high-resolution en face OCT angiogram of the retinal vasculature and multiple corresponding OCT B-scans and thus gives simultaneous viewing of both structural and blood flow information of the different levels of retina [15]. The vessel density (VD) and FAZ (foveal avascular zone) area are the OCTA parameters used for quantitative analysis of retinal microvasculature status [16]. There are many different OCTA devices that investigate the vascular status of the macula with different scan protocols, but the lack of any consensus and some drawbacks about the accuracy of measurements can interfere with routine clinical patient evaluations especially in distorted anatomy, such as DME [17]. Additionally, there are few studies to confirm the widespread clinical use of automated analysis built into software in commercially available devices.

Although the exact pathogenesis of DME has not been fully elucidated, we hypothesized that the integrity of the capillary plexuses in each quadrant changes differently and might be associated with structural changes in a sectoral manner in DME.

\section{Methods}

This was a retrospective cross-sectional single-center study. All patients were recruited at the Ophthalmology Department of Inonu University between October 2016 and December 2020. Signed informed consent was obtained from all patients. The study was approved by the local Ethical Committee and conducted in accordance with the Declaration of Helsinki.

Patients suffering from center-involved DME who had undergone OCTA examination were enrolled. The patients were $>18$ years old and had type II diabetes mellitus. Age- and sex-matched healthy subjects were included as a control group. Complete ophthalmologic examination of all patients was obtained from the records. Best-corrected visual acuity (BCVA) was recorded using Snellen charts and then converted into the logarithm of the minimum angle of resolution (logMAR) for statistical analysis. DME was defined as the presence of retinal thickening, intraretinal cyst, or subretinal fluid on B-scans. Central foveal thickness (CFT) greater than $270 \mu \mathrm{m}$ was included in the DME group. We included patients who received one or consecutive multiple injections with aflibercept, ranibizumab, bevacizumab or dexamethasone.

The criteria for exclusion were as follows: significant media opacity limiting the quality of OCTA images, OCTA images with poor quality $(<60)$, OCTA images with blinking artifacts or motion artifacts, patients with choroidal neovascularization (CNV), high myopia, retinal vein occlusion, uveitis, uncontrolled glaucoma, endophthalmitis, vitreomacular traction, epiretinal membrane, macular hole, macular scar, previous vitrectomy or any ocular surgery (except uneventful cataract surgery at least 3 months before the study), and macular grid laser. 


\section{OCT Angiography Imaging and Measurements}

All participants underwent OCTA using swept-source OCT (DRI OCT Triton; Topcon Inc, Tokyo, Japan) with a wavelength of $1050 \mathrm{~nm}$, an acquisition speed of 100000 A-scans per second, and axial and transversal resolutions of 8 and $20 \mathrm{~mm}$ in tissue, respectively. We used a $6 \times 6 \mathrm{~mm}$ volumetric scan centered at the fovea, each consisting of 512 A-scans per B-scan for a total of 512 B-scans, to obtain OCTA images. The device has an active eye tracker to reduce motion and blinking artifacts during OCTA. The OCTA images were obtained with a quality score of 60 and above. Sections of the SCP and DCP were automated and segmented by the built-in software IMAGEnet6, v1.25 and OCTARA ${ }^{\mathrm{TM}}$ (Topcon) software; the SCP was delineated by $2.6 \mathrm{~mm}$ below the internal limiting membrane to $15.6 \mathrm{~mm}$ below the junction between the inner plexiform and the inner nuclear layers; the DCP was delineated by $15.6 \mathrm{~mm}$ below the inner plexiform and the inner nuclear layers to $70.2 \mathrm{~mm}$ below them. The accuracy of the automatic segmentation lines was verified visually and independently by experienced graders (NP). Segmentation errors were manually corrected by a grader. If not, the subject was excluded from the study. Eyes with hemorrhage and hard exudates in the measurement area were excluded.

VD was described as the proportion of area occupied by vessels. The VD was measured using the instrument's built-in software based on the OCT angiography image intensity, and values were recorded for four different quadrants: the inferior superior, nasal and temporal (i, S, N, and T) parafovea (diameter $3 \mathrm{~mm}$ ) based on the ETDRS grid in both plexuses. The FAZ was defined as the area encompassing the central fovea, where there were no clear and demarcated vessels seen on OCTA. The FAZ area was manually contoured and measured using a device area measurement tool in both the SCP and DCP (Fig. 1). The presence of cysts was determined with en face OCTA imaging in a sectoral manner in both the SCP and DCP.

\section{Structural Measurements}

Structural measurements were performed on $9 \mathrm{~mm}$ width 12 radial scans that cover all sectors and allow sectoral analysis using swept-source OCT. CFT and sectoral parafoveal retinal thicknesses (RT) (i, S, N, and T) were quantified automatically with the instrument's built-in software in the $3 \mathrm{~mm}$ ETDRS grid macular map. The integrity of the ellipsoid zone (EZ) and external limiting membrane (ELM) were evaluated in all 12 radial B-scan images. The presence of ellipsoid zone disruption (EZD) and external limiting membrane disruption (ELMD) were defined as any disruption of the integrity of the EZ and ELM within the $3 \mathrm{~mm}$ ETDRS grid in a sectoral manner (i, S, N, and T). EZ disruption and ELM disruption were defined as any clear discontinuation in related lines within the $3 \mathrm{~mm}$ ETDRS grid (Fig. 2). The presence of cysts in the INL and OPL was recorded regardless of the number and size by examining radial sections in a sectoral manner (i, S, N, and T).

\section{Statistical analysis}

Study data were analyzed by using SPSS version 22.0 (IBM Corporation, Armonk, New York, USA). The conformity of univariate data to a normal distribution was evaluated with the Shapiro-Wilk test. Normally 
distributed quantitative variables are shown as the mean \pm standard deviation (SD) and median (minimum and maximum) when nonnormally distributed. The categorical variables are shown as $\mathrm{n}(\%)$ in tables. According to the results, the independent-samples $t$ test and Mann-Whitney $U$ test were used to compare two independent groups. Categorical variables were compared with Pearson's chi-square test or Fisher's exact test. Pearson's and Spearman's correlation tests were used to analyze the correlations of variables with each other. A p-value $<0.05$ was accepted as significant.

\section{Results}

Twenty-six eyes with DME and 20 eyes of the normal healthy subjects were analyzed. The baseline characteristics are reported in Table 1. No significant differences were observed between patients with DME and controls in age, sex, or eye distribution. BCVA was significantly different between the two groups $(p<0.0001)$. Of the 26 eyes included in the DME group, $22(84.6 \%)$ eyes had laser photocoagulation in a panretinal manner, and $23(88.5 \%)$ eyes had at least 1 intravitreal injection with bevacizumab, aflibercept, ranibizumab or dexamethasone implants.

Table 1

Baseline characteristics of the patients

\begin{tabular}{|llll|}
\hline & Group 1 (n: 26) & Group 2 (n: 20) & P \\
\hline $\begin{array}{l}\text { Age (mean } \pm \text { SD, median(min- } \\
\text { max)), years }\end{array}$ & $\begin{array}{l}59.08 \pm 6.41, \\
\text { Sex n(\%)(male) }\end{array}$ & $57.80 \pm 7.50,55(49-74)$ & 0.537 \\
& $16(61.5)$ & $12(60)$ & $0.916\left(\chi^{2}:\right.$ \\
\hline Eye distrubitions n(\%) (Right) & $16(61.5)$ & $12(60)$ & $0.11)$ \\
\hline $\begin{array}{l}\text { BCVA (mean } \pm \text { SD, median(min- } \\
\text { max)), logMar }\end{array}$ & $0.46 \pm 0.46$, & $0.004 \pm 0.013$, & $0.11)$ \\
\hline BCVA: Best-corrected visual acuity & $0.30(0.0-1.3)$ & $0.00(0.00-0.045)$ & $0.0001^{*}$ \\
\hline
\end{tabular}

\section{OCTA values}

Table 2 shows the corresponding OCTA measurements in the superficial and deep capillary plexuses in eyes with DME and controls. Sectoral analysis was performed to compare the VD between each group. In the SCP, there were no significant differences in inferior and temporal VD (P: 0.170 and P: 0.058), but superior and nasal VD was significantly lower in the DME eyes (P: 0.0001 and P: 0.020 , respectively). In the DCP, there were no significant differences in inferior, superior and nasal VD (P: 0.242, P: 0.070, and P: 0.251, respectively), but temporal VD was significantly lower in the DME eyes (P: 0.034) (Fig. 3). The VD in the inferior quadrant of the SCP was not correlated with the inferior quadrant of the DCP, and the VD in the superior quadrant of the SCP was not correlated with the superior quadrant of the DCP, and the VD in 
the nasal quadrant of the SCP was not correlated with the nasal quadrant of the DCP, the VD in the temporal quadrant of the SCP was not correlated with the temporal quadrant of the DCP (all P > 0.05 in the Pearson or Spearman correlation test). 
Table 2

OCTA values

\begin{tabular}{|c|c|c|c|}
\hline & Group 1 & Group 2 & $\mathbf{P}$ \\
\hline Inferior VD-SCP, mean \pm SD (\%) & $45.57 \pm 4.01$ & $47.03 \pm 2.76$ & 0.170 \\
\hline Süperior VD-SCP, mean $\pm \mathrm{SD}(\%)$ & $43.25 \pm 4.34$ & $47.85 \pm 2.42$ & $0.0001 *$ \\
\hline Nasal VD-SCP, mean \pm SD (\%) & $42.13 \pm 3.59$ & $44.32 \pm 2.14$ & $0.020 *$ \\
\hline $\begin{array}{l}\text { Temporal VD-SCP, mean } \pm \text { SD, } \\
\text { median(min-max) }(\%)\end{array}$ & $\begin{array}{l}43.02 \pm 7.66,43.83 \\
(10.18-51.01)\end{array}$ & $\begin{array}{l}45.93 \pm 1.86,45.85 \\
(42.81-49.45)\end{array}$ & 0.058 \\
\hline $\begin{array}{l}\text { FAZ area-SCP, median(min-max) } \\
\left(\mu \mathrm{m}^{2}\right)\end{array}$ & $367(168-805)$ & $240(125-549)$ & $0.003^{*}$ \\
\hline $\begin{array}{l}\text { The presence of cyst in inferior } \\
\text { SCP }(+ \text { eyes }) \%\end{array}$ & $16(61.5)$ & NA & \\
\hline $\begin{array}{l}\text { The presence of cyst in süperior } \\
\text { SCP(+ eyes)\% }\end{array}$ & $18(69.2)$ & NA & \\
\hline $\begin{array}{l}\text { The presence of cyst in nasal } \\
\text { SCP }(+ \text { eyes }) \%\end{array}$ & $12(46.2)$ & NA & \\
\hline $\begin{array}{l}\text { The presence of cyst in temporal } \\
\text { SCP(+ eyes)\% }\end{array}$ & $20(76.9)$ & NA & \\
\hline Inferior VD-DCP, mean $\pm \mathrm{SD}(\%)$ & $23.97 \pm 5.47$ & $25.63 \pm 3.42$ & 0.242 \\
\hline Süperior VD-DCP, mean \pm SD(\%) & $21.76 \pm 8.82$ & $25.59 \pm 3.03$ & 0.070 \\
\hline Nasal VD-DCP, mean \pm SD(\%) & $23.82 \pm 7.35$ & $25.88 \pm 3.22$ & 0.251 \\
\hline Temporal VD-DCP, mean \pm SD $(\%)$ & $19.76 \pm 6.92$ & $23.37 \pm 2.86$ & $0.034^{*}$ \\
\hline $\begin{array}{l}\text { FAZ area-DCP, median(min-max) } \\
(\mu \mathrm{m} 2)\end{array}$ & $852(214-4496)$ & $412(147-803)$ & $0.0001 *$ \\
\hline $\begin{array}{l}\text { The presence of cyst in inferior } \\
\text { DCP }(+ \text { eyes }) \%\end{array}$ & $21(80.8)$ & NA & \\
\hline $\begin{array}{l}\text { The presence of cyst in süperior } \\
\text { DCP(+ eyes)\% }\end{array}$ & $24(92.3)$ & NA & \\
\hline $\begin{array}{l}\text { The presence of cyst in nasal } \\
\text { DCP }(+ \text { eyes }) \%\end{array}$ & $20(76.9)$ & NA & \\
\hline $\begin{array}{l}\text { The presence of cyst in temporal } \\
\text { DCP(+ eyes)\% }\end{array}$ & $25(96.2)$ & NA & \\
\hline
\end{tabular}

The FAZ area was significantly greater (both superficial and deep) in DME eyes than in control eyes (P: 0.003 and P: 0,0001, respectively) (Fig. 4). There was no correlation between the FAZ area of the SCP and VD in any quadrant of the SCP (all P > 0.05 in the Pearson or Spearman correlation test), but the FAZ area 
of the DCP was negatively correlated with the nasal and temporal VD of the DCP (Spearman r: -0.467 P: 0.016 nasal, and r: -0. 742 P: 0.0001 temporal).

The sectoral analysis of the presence of cysts performed with En face OCT revealed that 16 eyes (61.5\%) had inferior, 18 eyes (69.2\%) superior, 12 eyes (46.2\%) nasal, and 20 eyes (76.9\%) temporal cysts in the area of the SCP. The VD in any of these quadrants was not affected by the presence of cysts in the corresponding quadrants of the SCP (all P > 0.05 in the Mann-Whitney U test). The FAZ area of the SCP was not affected by the presence of cysts in any quadrant of the SCP (all P > 0.05 in the Mann-Whitney $U$ test). The corresponding retinal quadrant thicknesses were affected by the cysts in the SCP, as expected (all $\mathrm{P}<0.05$ in the Mann-Whitney $\mathrm{U}$ test).

In the area of the DCP, 21 eyes (80.8\%) had inferior, 24 eyes (92.3\%) had superior, 20 eyes (76.9\%) had nasal, and 25 eyes (96.2\%) had temporal cysts in DME eyes. The VD in any of these quadrants was not affected by the presence of cysts in the corresponding quadrants of the DCP (all P>0.05 in the MannWhitney U test), but the VD in the nasal and temporal quadrants of the DCP was affected by the cysts in the corresponding SCP quadrants (P: 0.017 and P: 0.0.004, respectively; Mann-Whitney U test). The FAZ area of the DCP was not affected by the presence of cysts in any quadrant of the SCP but was affected by the presence of cysts only in the superior and nasal quadrants of the DCP (P: 0.012 and P: 0.0.028, respectively; Mann-Whitney $U$ test). The corresponding retinal quadrant thicknesses were affected by cysts in the DCP-only superior and nasal quadrants (P: 0.025 and P: 0.0.028, respectively; Mann-Whitney U test).

When considering the presence of cysts in the layers, cysts were detected in the INL in 20 eyes (76.9\%), and cysts were detected in the OPL in 22 eyes (84.6\%).

\section{Structural OCT analysis}

Structural analysis results are reported in Table 3. The CFT was significantly different between the two groups, as expected $(p<0.0001)$. The sectoral analysis of the thicknesses according to the $3 \mathrm{~mm}$ ETDRS grid sectors revealed that inferior, superior, nasal and temporal area thicknesses were significantly greater in DME eyes (P: 0.0001, P: 0.0001, P: 0.005, and P: 0.0001, respectively). When considering correlations between the VD in quadrants and sectoral retinal thicknesses in DME eyes, only the nasal quadrant VD in the SCP was positively correlated with nasal thickness (Spearman r: 0.413, P: 0.036). All VDs in the DCP were negatively correlated with thickness when the corresponding quadrants were evaluated (Spearman r: -0.113 P: 0. 583 inferior, r: -0.534 P: 0.005 superior, r: -0.470 P: 0.015 nasal, r: -0.667 P: 0.0001 temporal). 
Table 3

Structural OCT analysis

\begin{tabular}{|llll|}
\hline & Group 1 & Group 2 & P \\
\hline CFT median(min-max) $(\mu \mathrm{m})$ & $341(273-679)$ & $251(216-269)$ & $\mathbf{0 . 0 0 0 1 *}$ \\
\hline Inferior RT median(min-max) $(\mu \mathrm{m})$ & $346(289-564)$ & $308(278-337)$ & $\mathbf{0 . 0 0 0 1 *}$ \\
\hline Süperior RT median(min-max) $(\mu \mathrm{m})$ & $354(293-594)$ & $318(278-342)$ & $\mathbf{0 . 0 0 0 1 *}$ \\
\hline Nasal RT median(min-max) $(\mu \mathrm{m})$ & $346(291-529)$ & $315(275-344)$ & $\mathbf{0 . 0 0 5 *}$ \\
\hline Temporal RT (median(min-max) $\mu \mathrm{m})$ & $378(309-592)$ & $301(262-326)$ & $\mathbf{0 . 0 0 0 1 *}$ \\
\hline ELM disruption in inferior (+ eyes)\% & $6(23.1)$ & NA & \\
\hline ELM disruption in süperior (+ eyes)\% & $5(19.2)$ & NA & \\
\hline ELM disruption in nasal (+ eyes)\% & $5(19.2)$ & NA & \\
\hline ELM disruption in temporal (+ eyes)\% & $7(26.9)$ & NA & \\
\hline EZ disruption in inferior (+ eyes)\% & $11(42.3)$ & NA & \\
\hline EZ disruption in süperior (+ eyes)\% & $11(42.3)$ & NA & \\
\hline EZ disruption in nasal (+ eyes)\% & $10(38.5)$ & NA & NA \\
\hline EZ disruption in temporal (+ eyes)\% & $13(50)$ & N $)$ & \\
\hline CFT: Central foveal thickness, RT: Parafoveal retinal thickness, ELM: External limitan membrane, EZ: Ellipsoid zone \\
\hline
\end{tabular}

ELM disruptions detected in each quadrant were 6 eyes (23.1\%) inferior, 5 eyes (19.2\%) superior, 5 eyes (19.2\%) nasal and 7 eyes (26.9\%) in temporal scans. ELM disruptions were not affected by corresponding VDs in the SCP (all P > 0.05), but nasal and temporal quadrant ELM disruptions were affected by VDs in the DCP in the corresponding quadrants (P: 0.005 nasal, P: 0.001 temporal; Mann-Whitney U test). ELM disruptions in each quadrant were not affected by the FAZ area in the SCP (all P>0.05) but were affected by the FAZ area in the DCP (all $P<0.05$; Mann-Whitney $U$ test). ELM disruptions were affected by macular thicknesses in each corresponding quadrant (all $\mathrm{P}<0.05$; Mann-Whitney $\mathrm{U}$ test).

EZ disruptions detected in each quadrant were 11 eyes (42.3\%) inferior, 11 eyes (42.3\%) superior, 10 eyes (38.5\%) nasal and 13 eyes (50\%) in temporal scans in DME eyes. EZ disruptions were not affected by the corresponding VDs in the SCP and DCP (all P > 0.05; Mann-Whitney U test). EZ disruptions in each quadrant were not affected by the FAZ area in the SCP and DCP (all P >0.05). EZ disruptions were not affected by macular thicknesses in the inferior, superior and nasal corresponding quadrants (all $P>0.05)$ but were affected in the temporal quadrants (P: 0.034).

\section{Discussion}


The VD and FAZ area are OCTA parameters used for quantitative analysis of retinal microvasculature status [16]. The importance of the VD and FAZ on DME in the SCP and DCP has been confirmed in many studies $[14,18,19]$. Al of these studies investigated the macula globally. In the present study, we investigated vascular status and the association with structural morphology of the macula in a sectoral manner. A $3 \mathrm{~mm}$ ETDRS macular grid evaluation of our study revealed that the VD was significantly lower in the superior and nasal quadrants in the SCP and the temporal quadrant of the DCP in DME eyes compared to the corresponding quadrants in healthy eyes. The FAZ area was significantly greater (both superficial and deep) in DME eyes than in control eyes. The sectoral analysis of the thicknesses according to $3 \mathrm{~mm}$ ETDRS grid sectors revealed that inferior, superior, nasal and temporal quadrant thicknesses were significantly greater in DME eyes. ELM disruptions detected in each quadrant were 6 eyes $(23.1 \%)$ inferior, 5 eyes (19.2\%) superior, 5 eyes (19.2\%) nasal and 7 eyes (26.9\%) in temporal scans. EZ disruptions detected in each quadrant were 11 eyes (42.3\%) inferior, 11 eyes (42.3\%) superior, 10 eyes (38.5\%) nasal and 13 eyes (50\%) in temporal scans in DME eyes.

In diabetic eyes without clinically detectable DR, the VD of both superficial and deep capillary plexuses was lower than that in healthy controls, and eyes with a worse DR stage showed larger FAZ areas and lower VD values $[20,21]$. Moreover, the risk of DR progression was found to be associated with a larger FAZ area and lower VD on the DCP, whereas the risk of DME development was found to be associated with lower VD on the SCP $[18,22]$. There is growing evidence that the DCP is affected earlier than the SCP in DR because of the terminal architecture of the deep capillaries $[19,23]$. However, the rationality and priority of the SCP or DCP on the formation of the DME remain unclear $[18,19]$. To contribute to understanding the pathogenesis of DME, we conducted sectoral analysis and found that the VD was significantly lower in the superior and nasal quadrants in the SCP and temporal quadrant of the DCP in DME eyes. The VDs in the SCP appeared to be affected in more quadrants, although the decrease in the temporal quadrant of the SCP did not reach significance because of the small sample size (P: 0.058). The importance of our results may be that the SCP was affected more in advanced DR with DME. The SCP includes larger vessels (arterioles and venules) beside the capillaries. Additionally, it was found that the diameter of large vessels steadily increased from diabetes without retinopathy to more severe stages of diabetic retinopathy in SCP [24]. Vessel length and vessel caliber affect vessel density [25]. This outcome may result in a limited decrease in the VD, although capillary dropout occurs in the SCP. We assumed that our DME eyes had advanced DR because almost all had a history of Ifk and intravitreal injection. Our correlation analysis between the VD of the SCP and DCP revealed that there were no correlations in the corresponding quadrants. This result may indicate that the SCP and DCP are affected independently from each other. On the other hand, there is probably a compensatory relationship between the SCP and DCP; thus, there may be dependence [26]. We suggest that in DR, the DCP may first be affected but compensated by a relatively normal SCP to prevent the formation of macular edema. However, if the decrease in the VD in the SCP exceeded the compensation of the DCP in advanced DR (involvement in more quadrants, such as our results), DME occurred. To the best of our knowledge, such a finding has not been mentioned before in the literature. Longitudinal studies are needed to confirm these results. 
By the automated quantitative approach, VD calculations may confound because of cystoid spaces in the regions counted as nonperfused areas [27]. Many researchers have excluded these areas in the images to calculate the VD, but according to the results of the present study, we suggest that cystoid spaces actually occur in vascular rarefaction areas. Moreover, some studies on VD measurements found that the VD did not change after treatment, although anatomical relief was observed $[6,28]$. That finding supports our consideration. Nevertheless, pushed capillaries (vessel displacement) that are due to cystoid spaces may affect the calculation of the VD $[29,30]$. In our DME eyes, the VD in any of the SCP quadrants was not affected by the presence of cysts in the corresponding quadrants of the SCP or DCP. The VD in any of the DCP quadrants was not affected by the presence of cysts in the corresponding quadrants of the DCP, but the presence of cysts in the nasal and temporal quadrants of the SCP affected the VD in the corresponding quadrants of the DCP. This result may be due to the distortion effect of cysts in the SCP on the capillaries of the DCP. We did not measure the actual area of the cysts because it requires different image processing, and we only evaluated the presence of the cysts in the corresponding quadrants. Future studies that include the measurement of the actual area of cysts may provide a better explanation for this effect. Mané et al. found that cystoid spaces were often located within areas of nonperfusion, and cysts were more visible and more numerous in the deeper retina [7]. Consistent with that finding, the majority of our patients had cysts in the DCP, especially in the temporal quadrant, where there was a significant decrease in the VD, but the association could not be proven statistically. The actual area occupied by cysts may be meaningful for that association.

When considering correlations between the VD in quadrants and sectoral retinal thicknesses in DME eyes, all VDs in the DCP were negatively correlated with thicknesses, but only the nasal quadrant VD in the SCP was positively correlated with nasal thickness when the corresponding quadrants were evaluated. These findings may reflect that edematous thickening is especially linked with a decrease in the VD of the DCP, but we cannot say that there is the same link between the VD of the SCP and thickening because of the possible compensatory changes in the SCP, as mentioned above.

Enlargement of FAZ areas at the level of both the SCP and DCP in DR and diabetic maculopathy has been proven in many studies, consistent with our study $[5,21]$. In terms of the correlation between the FAZ area and sectoral VDs, there were no associations in the SCP, but the nasal and temporal quadrants of the DCP VDs were negatively correlated with the FAZ area of the DCP in the present study. A possible explanation for this finding may be that the FAZ area of the SCP increases because of edematous distortion. However, the increase in the FAZ area of the DCP may be due to capillary dropout, especially in the nasal and temporal quadrants of the DCP, in our patients. It is supposed that the accumulation of fluid in the macula causes distortion and enlargement of the FAZ area [23,31]. Normally, in the inner retinal layers, interstitial fluid is strictly controlled by the retinal capillary plexus and Müller cells. When fluid production exceeds fluid reabsorption, macular edema forms [29]. Excessive extravasation of fluid from the vascular bed to the interstitial space may cause the formation of cystoid spaces and tissue distortion [32]. We could not find any association between sectoral thicknesses and the FAZ area of the SCP, but the FAZ area of the DCP was correlated positively with both quadrants and central thicknesses. In our DME eyes, the FAZ area of the SCP was not affected by the presence of cysts in any quadrants of the SCP or DCP. 
The FAZ area of the DCP was not affected by the presence of cysts in any quadrant of the SCP but was affected by the presence of cysts only in the superior and nasal quadrants of the DCP. Again, this result may be due to the distortion effect of cysts on the capillaries in the DCP but nevertheless probably because of capillary dropout. We observed that the cysts in the DCP had greater dimensions, so a distortion effect of the cysts may be evident in the DCP. The expansion of the cystoid spaces may cause disruption of the wall of the cysts that contain Müller cells. Müller cells have important structural and functional duties. Müller cells that span from the internal limiting membrane (ILM) to the ELM provide the scaffold of the retinal layers. Müller cells wrap the retinal capillaries and have a tight relationship with the vascular bed functionally and morphologically. Therefore, it seems that the association between the presence of cysts and vascular status is a vicious circle.

Moon et al. showed the importance of the VD and FAZ areas of the DCP on the ELM and EZ [33]. Consistent with these findings, we found that ELM disruptions were not affected by the corresponding VDs in the SCP, but nasal and temporal quadrant ELM disruptions were affected by VDs in the DCP in the corresponding quadrants. ELM disruptions in each quadrant were not affected by the FAZ area in the SCP but were affected by the FAZ area in the DCP. In our sectoral analysis, we could not find any association between EZ disruptions and the VD of either the SCP or DCP when the corresponding quadrants were evaluated. Again, we did not find any association between EZ disruptions and the FAZ area of either the SCP or DCP. All these results may confirm that ELM is primarily affected by the DCP, especially in the nasal and temporal quadrants, but that cannot be said for EZ. The presence of EZ disruption was approximately two times more frequent according to ELM disruption in our subjects. In a recent study, Lei et al. reported that EZ disruptions were found in $40 \%$ of diabetic eyes [24]. Our sectoral results were comparable, but their subjects had $57 \%$ DME, while all of eyes in our study group had DME. Therefore, their results are relatively higher according to ours. That may be due to devices used. They used SD-OCT; however, we used SS-OCT, which was confirmed to have better deep resolution. Some researchers suggested that outer retinal disruptions were correlated with SCP changes, while some reported DCP partially contributed $[13,14,24]$. DCP provides approximately $15 \%$ of the oxygen supply of the photoreceptors, while the outer one-third of the retina is mainly supplied by the choriocapillaris [34]. On the other hand, Borrelli et al. reported an association between the EZ and the choriocapillaris [35]. Therefore, the EZ may be mainly dependent on the choriocapillaris rather than inner retinal capillary plexuses. Evaluation of the choriocapillaris was not within the scope of this study, and further studies are warranted to obtain more detailed information about the association between the EZ and choriocapillaris.

There are some limitations in this study. 1) This was a retrospective cross-sectional study, which may have caused ascertainment bias. 2) The small sample size was due to the strict inclusion and exclusion criteria; poor quality of the images and the presence of epiretinal membrane were the most common exclusion reasons. 3) The inclusion of both patients with differing previous treatments and treatmentnaive DME patients with different durations. 4) We performed all measurements on $6 \times 6 \mathrm{~mm}$ scans because we imaged all patients in a standard manner with a $6 \times 6 \mathrm{~mm}$ scan due to automated sectoral calculations in our clinical settings. If the $3 \times 3 \mathrm{~mm}$ scans give automated sectoral analysis, they may provide better measurements [36]. On the other hand, the suspended scattering particles in motion 
(SSPiM) may cause an overestimation of DCP vessel density in eyes with DME when 3-mm OCTA scans are used for analysis, but that effect may be less when 6-mm scans are used [37]. For these reasons, we used a $6 \mathrm{~mm}$ scan as the standard in all our patients, even for $3 \mathrm{~mm}$ area calculations. 5) The quantification structural analysis and the area of the FAZ may be affected by subjective judgment. For these reasons, all image processing was performed by the same person to reduce the interrater bias. It was stated previously that age and sex were correlated with both FAZ and VD of the SCP and DCP layer in both diabetic and healthy subjects [20]. We created the control group with the same age and sex distribution to eliminate these effects.

In conclusion, The VDs in the SCP appeared to be affected in different quadrants according to the DCP in advanced DR with DME. There were no correlations in the corresponding quadrants between the SCP and DCP in terms of VD. This result may indicate that the SCP was affected in more quadrants than the DCP and probably independently from each other. There was no correlation between the FAZ area and sectoral VDs in the SCP, but the nasal and temporal quadrants of the DCP VDs were negatively correlated with the FAZ area of the DCP. The presence of cysts may not be related to the vascular status of the SCP, probably due to large vessels in the SCP, but may be partially related to the vascular status of the DCP. ELM disruptions were associated with the vascular status of DCP but not SCP. EZ disruptions were not associated with the vascular status of the SCP and DCP. These results may confirm that the ELM is primarily affected by DCP, especially in the nasal and temporal quadrants, but that cannot be said for the EZ. Longitudinal studies are needed.

\section{Declarations}

\section{Funding:}

No funds, grants, or other support was received.

\section{Financial interests:}

The authors declare they have no financial interests.

\section{Conflicts of interest/Competing interests:}

No conflicting relationship exists for any author.

\section{Authors' contributions:}

All authors contributed to the study conception and design. Material preparation, data collection and analysis were performed by Nihat Polat and Saim Yologlu. The first draft of the manuscript was written 
by Nihat Polat and all authors commented on previous versions of the manuscript. All authors read and approved the final manuscript.

\section{Ethics approval:}

The study was approved by the local (Malatya clinic) Ethical Committee and conducted in accordance with the Declaration of Helsinki.

\section{Consent to participate:}

Informed consent was obtained from all individual participants included in the study.

\section{Consent for publication:}

Additional informed consent was obtained from all individual participants for whom identifying information is included in this article.

\section{References}

1. Sakata K, Funatsu H, Harino S et al (2007) Relationship of macular microcirculation and retinal thickness with visual acuity in diabetic macular edema. Ophthalmology 114(11):2061-2069. doi:10.1016/j.ophtha.2007.01.003

2. Diabetic Retinopathy Clinical Research Network, Wells JA, Glassman AR et al (2015) Aflibercept, bevacizumab, or ranibizumab for diabetic macular edema. N Engl J Med 372(13): 1193-1203. doi:10.1056/NEJMoa1414264

3. Arend O, Wolf S, Jung F et al (1991) Retinal microcirculation in patients with diabetes mellitus: dynamic and morphological analysis of perifoveal capillary network. Br J Ophthalmol 75(9):514518. doi:10.1136/bjo.75.9.514

4. Park JJ, Chung CS, Fawzi AA (2018) Visualizing Structure and Vascular Interactions: Macular Nonperfusion in Three Capillary Plexuses. Ophthalmic Surg Lasers Imaging Retina 49(11):e182e190. doi:10.3928/23258160-20181101-16

5. Hwang TS, Gao SS, Liu L et al (2016) Automated quantification of capillary nonperfusion using optical coherence tomography angiography in diabetic retinopathy. JAMA Ophthalmol 134:367-373. doi:10.1001/jamaophthalmol.2015.5658

6. Ghasemi Falavarjani K, lafe NA, Hubschman JP et al (2017) Optical coherence tomography angiography analysis of the foveal avascular zone and macular vessel density after anti- VEGF therapy in eyes with diabetic macular edema and retinal vein occlusion. Invest Ophthalmol Vis Sci 58:30-34. doi:10.1167/iovs.16-20579 
7. Mane V, Dupas B, Gaudric A et al (2016) Correlation between cystoid spaces in chronic diabetic macular edema and capillary nonperfusion detected by optical coherence tomography angiography. Retina 36(Suppl 1):102-110. doi:10.1097/IAE.0000000000001289

8. Sim DA, Keane PA, Fung S, Karampelas M, Sadda SR, Fruttiger M, Patel PJ, Tufail A, Egan CA (2014) Quantitative analysis of diabetic macular ischemia using optical coherence tomography. Invest Ophthalmol Vis Sci 55:417-423. doi:10.1167/iovs.13-12677

9. Tso MO (1982) Pathology of cystoid macular edema. Ophthalmology 89:902-915. doi:10.1016/s0161-6420(82)34698-9

10. Yanoff M, Fine BS, Brucker AJ, Eagle RC Jr (1984) Pathology of human cystoid macular edema. Surv Ophthalmol 28(suppl):505-511. doi:10.1016/0039-6257(84)90233-9

11. Yalçın NG, Özdek Ş (2019) The Relationship Between Macular Cyst Formation and Ischemia in Diabetic Macular Edema. Turk J Ophthalmol 49:194-200. doi:10.4274/tjo.galenos.2018.19616

12. Gao W, Tátrai E, Ölvedy V et al (2011) Investigation of changes in thickness and reflectivity from layered retinal structures of healthy and diabetic eyes with optical coherence tomography. J Biomed Sci Eng 04:657-665. doi:10.4236/jbise.2011.410082

13. Scarinci F, Jampol LM, Linsenmeier RA, Fawzi AA (2015) Association of diabetic macular nonperfusion with outer retinal disruption on optical coherence tomography. JAMA Ophthalmol 133(9):1036-1044. doi:10.1001/jamaophthalmol.2015.2183

14. Scarinci F, Nesper PL, Fawzi AA (2016) Deep retinal capillary nonperfusion is associated with photoreceptor disruption in diabetic macular ischemia. Am J Ophthalmol 168:129-138. doi:10.1016/j.ajo.2016.05.002

15. de Carlo TE, Romano A, Waheed NK et al (2015) A review of optical coherence tomography angiography (OCTA). Int J Retin Vitr 1:5. doi:10.1186/s40942-015-0005-8

16. Chu Z, Lin J, Gao C, Xin C, Zhang Q, Chen CL et al (2016) Quantitative assessment of the retinal microvasculature using optical coherence tomography angiography. J Biomed Opt 21:66008. doi:10.1117/1.JBO.21.6.066008

17. Corvi F, Pellegrini M, Erba S, Cozzi M, Staurenghi G, Giani A (2018) Reproducibility of Vessel Density, Fractal Dimension, and Foveal Avascular Zone Using 7 Different Optical Coherence Tomography Angiography Devices. Am J Ophthalmol 186:25-31. doi:10.1016/j.ajo.2017.11.011

18. Sun Z, Tang F, Wong R et al (2019) OCT angiography metrics predict progression of diabetic retinopathy and development of diabetic macular edema: a prospective study. Ophthalmology 126:1675-1684. doi:10.1016/j.ophtha.2019.06.016

19. Lee J, Moon BG, Cho AR, Yoon YH (2016) Optical Coherence Tomography Angiography of DME and Its Association with Anti-VEGF Treatment Response. Ophthalmology 123(11):2368-2375. doi:10.1016/j.ophtha.2016.07.010

20. Dimitrova G, Chihara E, Takahashi H et al (2017) Quantitative retinal optical coherence tomography angiography in patients with diabetes without diabetic retinopathy. Invest Ophthalmol Vis Sci 58:190-196. doi:10.1167/iovs.16-20531 
21. Samara WA, Shahlaee A, Adam MK et al (2017) Quantification of Diabetic Macular Ischemia Using Optical Coherence Tomography Angiography and Its Relationship with Visual Acuity. Ophthalmology 124(2):235-244. doi:10.1016/j.ophtha.2016.10.008

22. Tsai ASH, Gan ATL, Ting DSW et al (2020) Diabetic macular ischemia: Correlation of Retinal Vasculature Changes by Optical Coherence Tomography Angiography and Functional Deficit. Retina 40(11):2184-2190. doi:10.1097/IAE.0000000000002721

23. Sambhav K, Abu-Amero KK, Chalam KV (2017) Deep Capillary Macular Perfusion Indices Obtained with OCT Angiography Correlate with Degree of Nonproliferative Diabetic Retinopathy. Eur J Ophthalmol 27(6):716-729. doi:10.5301/ejo.5000948

24. Lei J, Xu X, Chen L, Abdelfattah NS (2021) Dilated retinal large vessels and capillaries associated with diabetic macular edema and photoreceptor loss respectively. Graefes Arch Clin Exp Ophthalmol Jan 8. doi: 10.1007/s00417-020-05039-2. Online ahead of print

25. Trinh M, Kalloniatis M, Nivison-Smith L (2019) Vascular changes in intermediate age-related macular degeneration quantified using optical coherence tomography angiography. Transl Vis Sci Technol 8:20. doi:10.1167/tvst.8.4.20

26. Hsiao CC, Yang CM, Chang-Hao Yang CH et al (2020) Correlations between visual acuity and macular microvasculature quantified with optical coherence tomography angiography in diabetic macular oedema. Eye 34:544-552. doi:10.1038/s41433-019-0549-1

27. de Carlo TE, Chin AT, Joseph T et al (2016) Distinguishing Diabetic Macular Edema From Capillary Nonperfusion Using Optical Coherence Tomography Angiography. Ophthalmic Surg Lasers Imaging Retina 47(2):108-114. doi:10.3928/23258160-20160126-02

28. Sorour OA, Sabrosa AS, Alibhai AY et al (2019) Optical coherence tomography angiography analysis of macular vessel density before and after anti-VEGF therapy in eyes with diabetic retinopathy. Int Ophthalmol 39:2361-2371. doi:10.1007/s10792-019-01076-x

29. Spaide RF (2016) Retinal vascular cystoid macular edema: Review and New Theory. Retina 36(10):1823-1842. doi:10.1097/IAE.0000000000001158

30. Spaide RF (2015) Volume-rendered optical coherence tomography of diabetic retinopathy pilot study. Am J Ophthalmol 160:1200-1210. doi:10.1016/j.ajo.2015.09.010

31. Murakami T, Nishijima K, Sakamoto A et al (2011) Foveal Cystoid Spaces Are Associated with Enlarged Foveal Avascular Zone and Microaneurysms in Diabetic Macular Edema. Ophthalmology 118:359-367. doi:10.1016/j.ophtha.2010.03.035

32. Govetto A, Sarraf D, Hubschman JP et al (2020) Distinctive Mechanisms and Patterns of Exudative Versus Tractional Intraretinal Cystoid Spaces as Seen With Multimodal Imaging. Am J Ophthalmol 212:43-56. doi:10.1016/j.ajo.2019.12.010

33. Moon BG, Um T, Lee J, Yoon YH (2018) Correlation between Deep Capillary Plexus Perfusion and Long-Term Photoreceptor Recovery after Diabetic Macular Edema Treatment. Ophthalmology Retina 2:235-243. doi:10.1016/j.oret.2017.07.003 
34. Birol G, Wang S, Budzynski E et al (2007) Oxygen distribution and consumption in the macaque retina. Am J Physiol Heart Circ Physio 293:H1696-H1704. doi:10.1152/ajpheart.00221.2007

35. Borrelli E, PalmieriM, Viggiano P et al (2020) Photoreceptor damage in diabetic choroidopathy. Retina 40:1062-1069. doi:10.1097/IAE.0000000000002538

36. Hirano T, Kitahara J, Toriyama Y et al (2019) Quantifying vascular density and morphology using different swept-source optical coherence tomography angiographic scan patterns in diabetic retinopathy. Br J Ophthalmol 103:216-221. doi:10.1136/bjophthalmol-2018-311942

37. Maltsev DS, Kulikov AN, Kazak AA, Bailey Freund K (2020) Suspended Scattering Particles in Motion May Influence Optical Coherence Tomography Angiography Vessel Density Metrics in Eyes with Diabetic Macular Edema. Retina doi: 10.1097/IAE.0000000000003016 Publish Ahead of Print

\section{Figures}

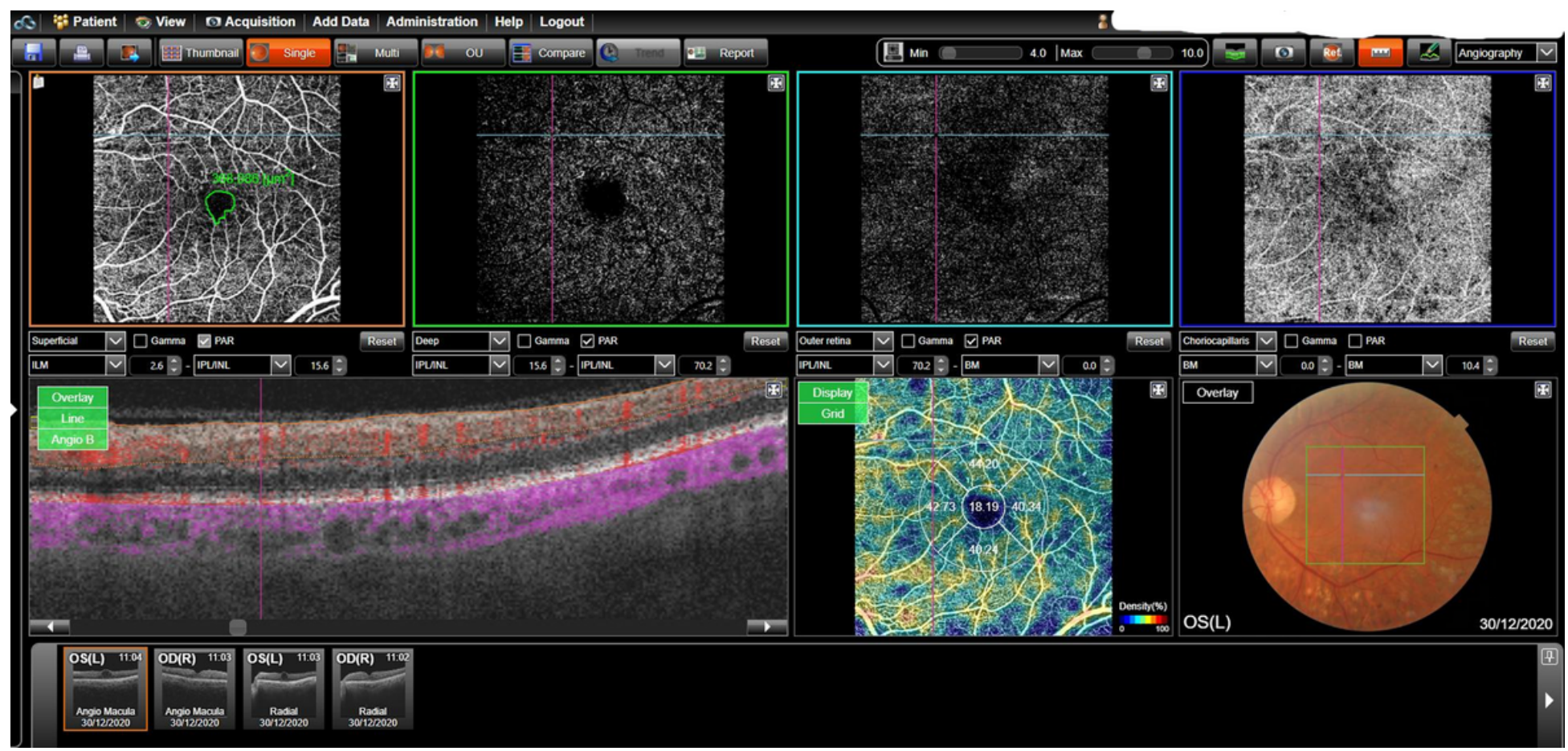

Figure 1

Quantification of retinal microvasculature from $6 \times 6 \mathrm{~mm}$ OCT angiography (OCTA) images of the superficial capillary plexus (SCP) and deep capillary plexus (DCP). Sectoral Vascular density (VD) measurement. The fovea was considered the central $1 \mathrm{~mm}$ diameter circle, and the parafovea was considered the area between the central circle and outer $3 \mathrm{~mm}$ diameter of the ETDRS circle. The VD of the parafoveal 3-mm zone was automatically measured by the software in inferior, superior, nasal and temporal quadrants (bottom row middle image). Foveal avascular zone (FAZ) area measurement. The FAZ area was manually contoured and measured using a device area measurement tool in both the SCP and DCP (top row left image) ETDRS: Early treatment of diabetic retinopathy study, 


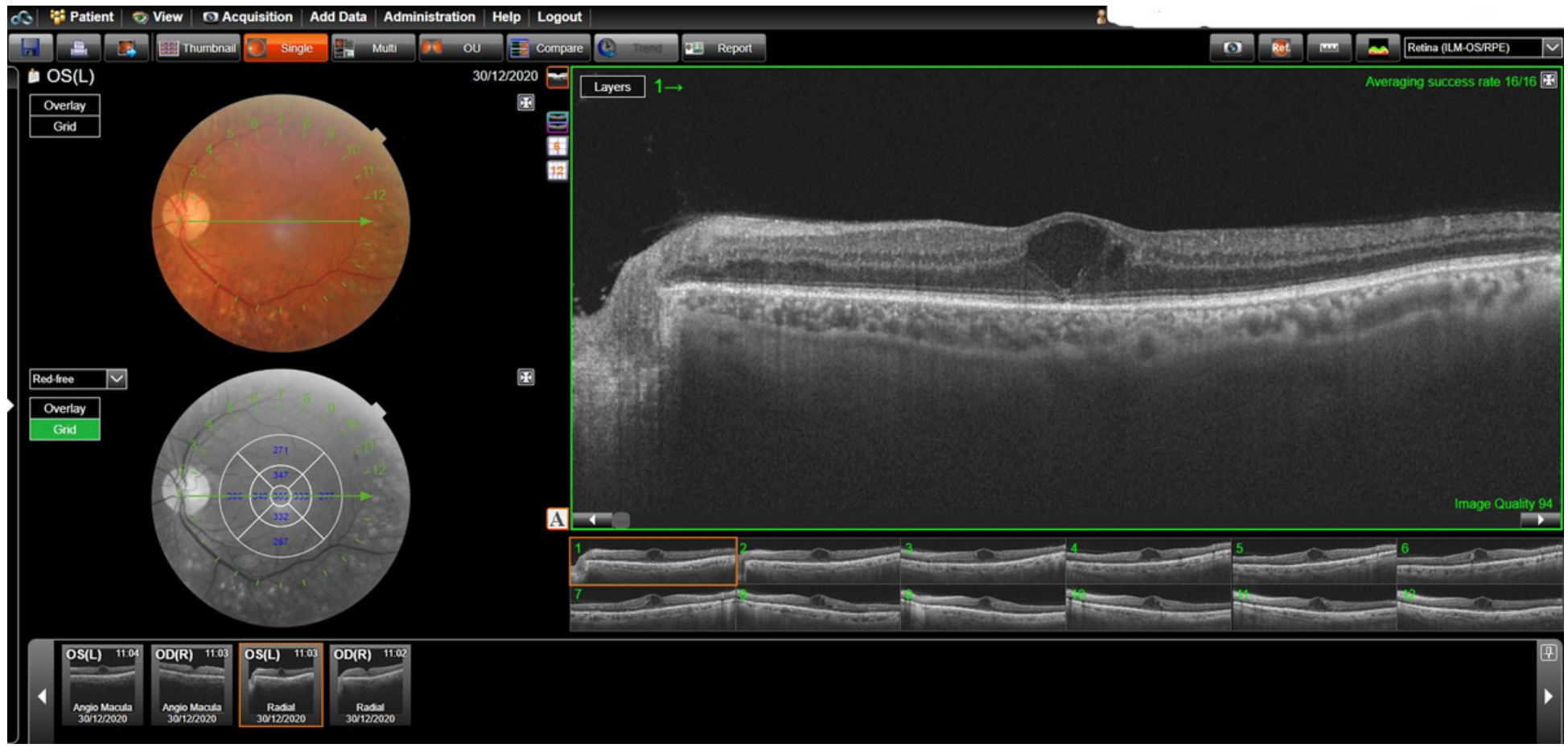

\section{Figure 2}

Sectoral retinal thicknesses (bottom left image). The integrity of the ellipsoid zone (EZ) and external limitan membrane (ELM) were evaluated in all 12 radial B-scan images (right images) 


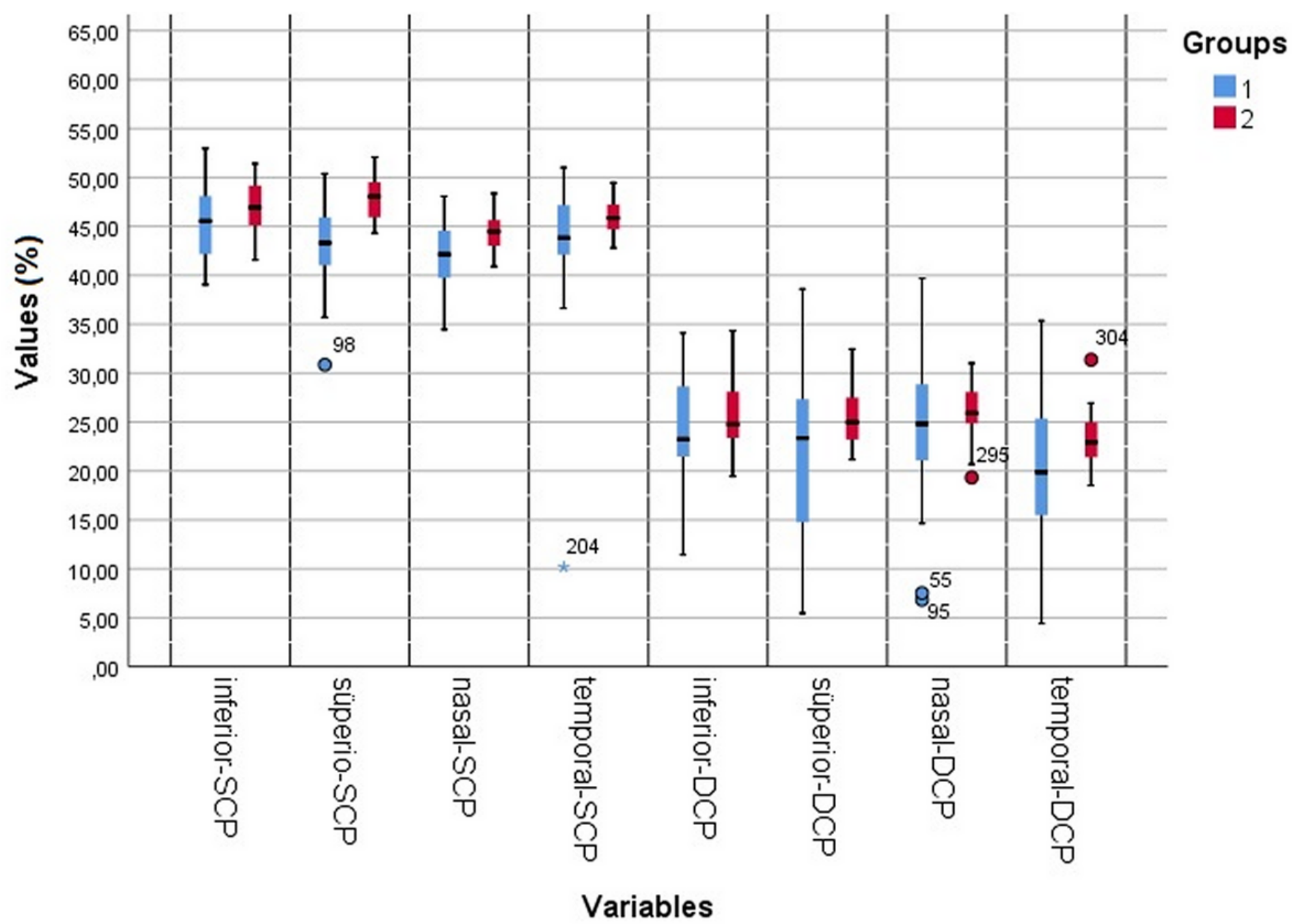

Figure 3

Sectoral Vascular density (VD) results of two groups 


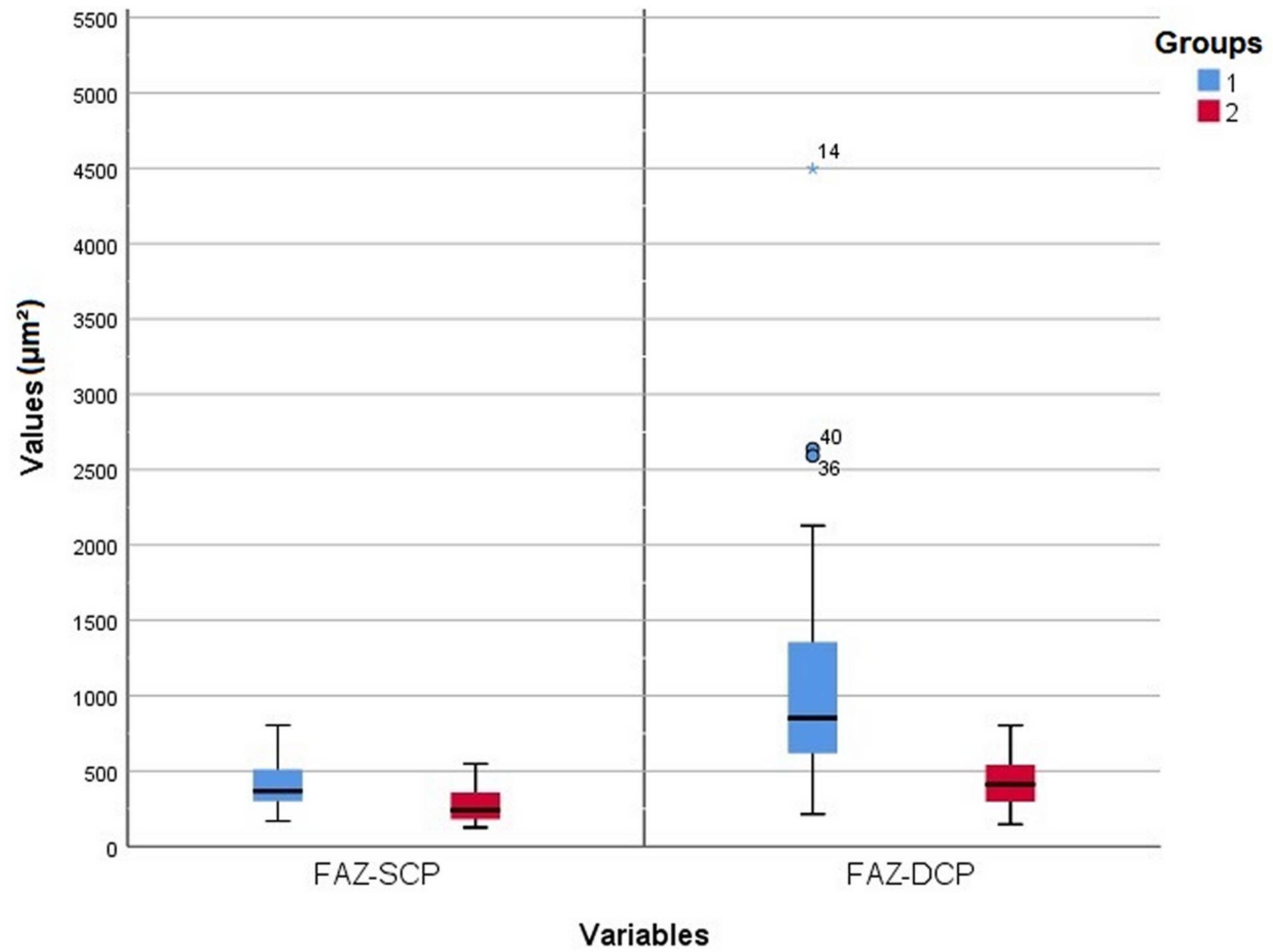

Figure 4

The FAZ areas of SCP and DCP in both groups 